\title{
Spin-polarized semiconductor surface states localized in subsurface layers
}

\section{$\operatorname{AUTHOR}(\mathrm{S})$ :}

Ohtsubo, Yoshiyuki; Hatta, Shinichiro; Yaji, Koichiro; Okuyama, Hiroshi; Miyamoto, Koji; Okuda, Taichi; Kimura, Akio; Namatame, Hirofumi; Taniguchi, Masaki; Aruga, Tetsuya

\section{CITATION:}

Ohtsubo, Yoshiyuki ...[et al]. Spin-polarized semiconductor surface states localized in subsurface layers. Physical Review B 2010, 82(20): 201307(R).

\section{ISSUE DATE:}

2010-11

\section{URL:}

http://hdl.handle.net/2433/134560

\section{RIGHT:}

(C) 2010 The American Physical Society 


\title{
Spin-polarized semiconductor surface states localized in subsurface layers
}

\author{
Yoshiyuki Ohtsubo, ${ }^{1,2}$ Shinichiro Hatta,,${ }^{1,2}$ Koichiro Yaji,${ }^{1,2}$ Hiroshi Okuyama, ${ }^{1}$ Koji Miyamoto, ${ }^{3}$ Taichi Okuda, ${ }^{3}$ \\ Akio Kimura, ${ }^{4}$ Hirofumi Namatame, ${ }^{3}$ Masaki Taniguchi, ${ }^{3,4}$ and Tetsuya Aruga ${ }^{1,2, *}$ \\ ${ }^{1}$ Department of Chemistry, Graduate School of Science, Kyoto University, Kyoto 606-8502, Japan \\ ${ }^{2}$ CREST, JST, Saitama 332-0012, Japan \\ ${ }^{3}$ Hiroshima Synchrotron Radiation Center, Hiroshima University, Higashi-Hiroshima 739-0046, Japan \\ ${ }^{4}$ Graduate School of Science, Hiroshima University, Higashi-Hiroshima 739-8526, Japan
}

(Received 15 October 2010; published 11 November 2010)

\begin{abstract}
A pair of different surface-state and surface-resonance bands has been identified on $\mathrm{Bi} / \mathrm{Ge}(111)$ $(\sqrt{3} \times \sqrt{3}) R 30^{\circ}$ by a combined experimental and computational study. The wave functions of the states have negligible amplitude at $\mathrm{Bi}$ atoms and are extended over more than 20 subsurface layers. These bands exhibit characteristic spin structure, which is ascribed to the combined Rashba and atomic spin-orbit interaction (SOI). Unlike previously known surface Rashba systems, the spin polarization is induced by SOI of a light element (Ge) with negligible contribution of a heavier one $(\mathrm{Bi})$.
\end{abstract}

DOI: 10.1103/PhysRevB.82.201307

PACS number(s): 73.20.At, 71.70.Ej

The space inversion asymmetry at surfaces lifts the spin degeneracy of surface states due to the Rashba spin-orbit interaction (SOI) ${ }^{1,2}$ Such spin polarization was first found on metal surfaces such as $\mathrm{Au}(111),{ }^{3,4}$ low-index surfaces of $\mathrm{Bi},{ }^{5}$ and Bi/Ag. ${ }^{6,7}$ Recently, the Rashba spin splitting has been reported also on semiconductor surfaces, such as $\mathrm{Si}(111)$ and $\mathrm{Ge}(111)$ covered with a monolayer of sixth-row elements, ${ }^{8-10}$ and is of emerging interest with respect to possible application to spin transport. ${ }^{11,12}$ The Rashba spin splitting observed to date are limited to the surfaces containing the sixth-row elements with the only exception of $\mathrm{Sb}(111),{ }^{13}$ in agreement with the common understanding that the large Rashba spin splitting is due to strong SOI at close proximity to nuclei of heavier atoms. ${ }^{5,14}$

In this Rapid Communication, we report on the electronic structure of Bi-covered $\mathrm{Ge}(111)$ studied by using angleresolved photoelectron spectroscopy (ARPES), spin-resolved ARPES (SARPES), and first-principles calculation. The result indicates that a spin-polarized surface state and a surface resonance are formed near the upper edge of the bulk valence band (VB) around $\bar{\Gamma}$. The first-principles calculation shows that the characteristic spin structure is induced by combined Rashba and atomic SOI. The wave functions of these states have a small or negligible amplitude at the topmost Bi layer but are spread over subsurface Ge layers. The results imply that the Bi SOI plays negligible role in the spin polarization and evidence the first experimental observation of a surfacestate spin polarization due to SOI of a fourth-row element.

ARPES and SARPES measurements were performed with energy resolutions of $\sim 10 \mathrm{meV}$ and $\sim 110 \mathrm{meV}$, respectively, by using He resonance lamps. The Fermi level $\left(E_{F}\right)$ was determined by photoemission from Mo sample holders in contact with the samples. We used $p$-type Ge(111) substrates $(0.2 \Omega \mathrm{cm})$ and prepared the $(\sqrt{3} \times \sqrt{3}) R 30^{\circ}$-Bi surface as prescribed elsewhere. ${ }^{9,15}$ All-electron full-potential band calculation was done by using the "augmented plane wave+local orbitals" method implemented in the WIEN2K code $^{16}$ with SOI taken into account. The surface was modeled by asymmetric slabs of $10,22,32$, and 44 Ge layers with one side covered with a $(\sqrt{3} \times \sqrt{3}) R 30^{\circ}$-Bi monolayer, the structure of which was optimized down to the tenth Ge layer starting from that determined by dynamical low-energy electron-diffraction analysis ${ }^{15}$ and the other side terminated by $\mathrm{H}$ atoms.

ARPES spectra measured near $\bar{\Gamma}$ along $\bar{\Gamma} \bar{M}$ are shown by solid lines in Fig. 1(a). The bulk VB maximum (VBM) is determined to be $0.11 \mathrm{eV}$ below $E_{\mathrm{F}}$ by using bulk Ge $3 d$ photoemission as a reference. ${ }^{17}$ The peak at the lowest binding energy, denoted $S$ in Fig. 1(a), is located at $80 \mathrm{meV}$ at $\bar{\Gamma}\left(\theta_{e}=0^{\circ}\right)$ and disperses downward at $\left|\theta_{e}\right| \geq 1^{\circ}$. As the peak $S$ is located in the projected bulk band gap, we identify this as a surface state.

The Mott detector used is equipped with four electron detectors in two orthogonal scattering planes. Two of the detectors are sensitive to the spin polarization perpendicular to $\bar{\Gamma} \bar{M}(U$ and $D)$, which is expected for usual Rashba SOI and the others almost parallel to $\bar{\Gamma} \bar{M}(L$ and $R$ ) under the geometry employed [Fig. 1(e)]. The SARPES spectra from the detectors for $U$ and $D$ are shown by triangles in Fig. 1(a). In the spectra at $\theta_{e}= \pm 1^{\circ}$ and $\pm 2^{\circ}$, two features spin polarized in opposite directions are evident at around 100 and 200 $\mathrm{meV}$. We subtracted a smooth polynomial background determined from the total signal of four detectors, which yielded the spectra shown in Fig. 1(b). Although the SARPES spectra are broader than the ARPES ones, the feature corresponding to $S$ is well discernible. While the $U$ and $D$ spectra coincide with each other at $\theta_{e}=0^{\circ}$, the $S$ band is spin polarized at $\theta_{e} \neq 0^{\circ}$ toward $U(D)$ at $\theta_{e}<0^{\circ}\left(\theta_{e}>0^{\circ}\right)$. The spectra in Fig. 1(b) indicate another component, labeled $S^{\prime}$, at 180-210 meV below $E_{\mathrm{F}}$. The $S^{\prime}$ state appears to be spin polarized toward the direction opposite to that of $S$. Note that the $S^{\prime}$ photoemission exhibits a small cross section in ARPES [Fig. 1(a)] due to partially polarized light used in ARPES and the geometry different from that for SARPES.

SARPES spectra for the $L / R$ polarization are shown in Fig. 1(c). The spin-independent background, which is the same as that used in Fig. 1(b), was subtracted [Fig. 1(d)]. Since the spin polarization probed in $L / R$ is parallel to initial-state electron wave vector $k_{\|}$, no Rashba-SOI-induced spin asymmetry along $L / R$ is expected for surface states. 

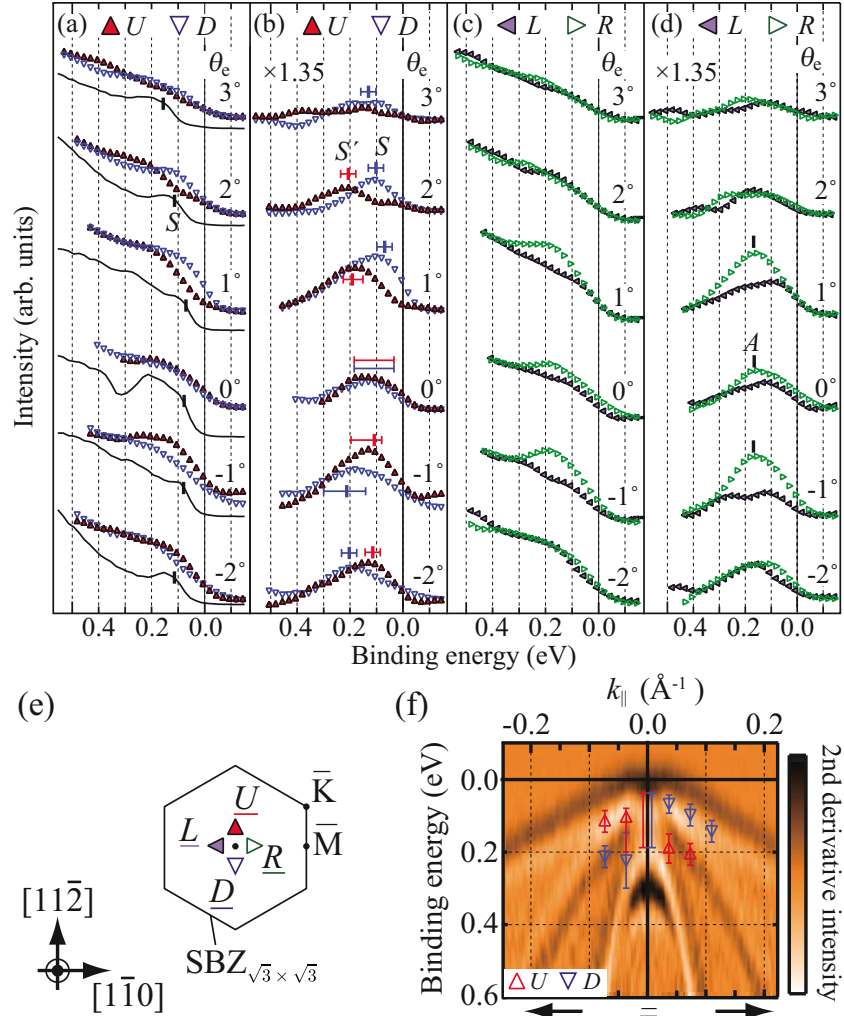

(f)

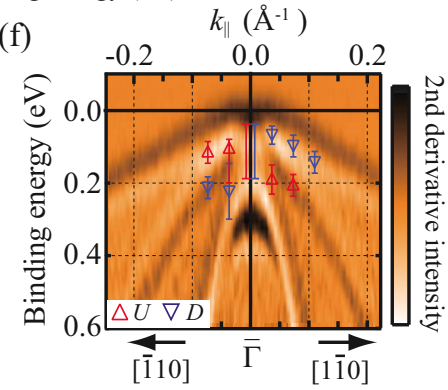

FIG. 1. (Color online) (a) ARPES spectra (solid lines) measured at room temperature with $\mathrm{He}$ I and SARPES spectra (triangles) measured at $100 \mathrm{~K}$ with $\mathrm{He} \mathrm{I}$ with spin polarization $U$ and $D$. (c) SARPES spectra with spin polarization $L$ and $R$. The common intensity scale is used for (a) and (c). [(b) and (d)] SARPES spectra in (a) and (c) from which the common, spin-independent polynomial background is subtracted and enlarged by a factor of 1.35. (e) Definition of the direction of spin polarization. (f) Second-derivative ARPES image (grayscale) and SARPES peak positions for $S$ and $S^{\prime}$.

However, the SARPES spectra in Fig. 1(c) indicate that a component $A$ with spin polarization toward $R$ exists at 160 meV below $E_{\mathrm{F}}$ within $\left|\theta_{e}\right| \leq 1^{\circ}$.

The $\bar{\Gamma}$ point in the surface Brillouin zone of the $(\sqrt{3}$ $\times \sqrt{3}) R 30^{\circ}$-Bi surface has the $C_{3 v}$ symmetry. Since the photoelectron wave function must be totally symmetric, the irreducible representation of possible final states is limited to $\Lambda_{6}$ of double group $C_{3 v}$. On the other hand, feature $A$ is located near the VBM of bulk Ge. The calculated band structure of bulk Ge is shown in Fig. 2. The highest occupied band of bulk Ge belongs to $\Lambda_{4+5} \cdot{ }^{18}$ An umklapp process involving the $(1 \times 1)$ surface reciprocal-lattice vector enables the transition from the $\Lambda_{4+5}$ state near $\Gamma$ to a $\Lambda_{6}$ final state at $\frac{2}{3} \Gamma L$, as shown in Fig. 2. For the excitation from the occupied $\Lambda_{4+5}$ state to the unoccupied $\Lambda_{6}$ final state, the off-normal incidence of unpolarized light induces the in-plane spin polarization. ${ }^{19}$ Taking our experimental geometry into account, the photoelectrons from the $\Lambda_{4+5}$ initial state are expected to be spin polarized toward $L / R$ but not toward $U / D .{ }^{20}$ The spectra in Fig. 1(d) suggest that the $A$ state gives a predominant contribution to the $R$ polarization and a much
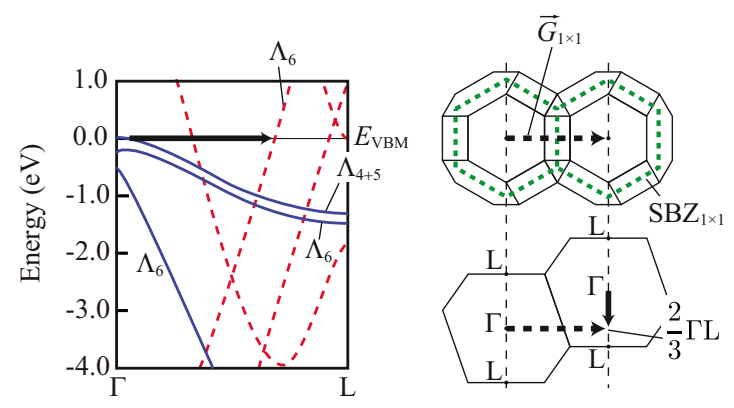

FIG. 2. (Color online) (Left) The calculated band structure of bulk Ge along $\Gamma L\left(k_{\|}=0\right)$. Solid (dashed) lines represent occupied (unoccupied) states. Unoccupied states are shifted downwards in energy by $20.2 \mathrm{eV}$, which corresponds closely to the photon energy minus the band gap underestimated by the calculation. (Right) The surface reciprocal-lattice vectors (dashed arrows) and bulk and surface Brillouin zones. The solid arrow represents the umklapp process observed.

smaller contribution, if exist, to the $L$ polarization. Since no spin polarization of photoelectrons is expected for the photoemission from the $\Lambda_{6}$ bulk band just below the $\Lambda_{4+5}$ band, we attribute $A$ to the photoemission from the bulk $\Lambda_{4+5}$ state at $k$ close to $\Gamma$. Note that the spin polarization is induced in the photoemission process, and the initial state is not spin polarized.

Let us now examine the spectra for $U / D$ in detail. For the spectra at $\left|\theta_{e}\right| \geq 2^{\circ}$, the peak positions are determined as shown by the tick marks in Fig. 1(b). For the spectra at $\theta_{e}$ $\leq 1^{\circ}$ the bulk component $A$ contributes to the $U / D$ spectra as "spin-degenerate" background. Note that, while the $S^{\prime}$ peak is located close in energy to $A$, the $S$ peak is higher by $\sim 100$ meV than $A$ and hence the peak position may be less affected. In order to determine the peak positions for $S$ and $S^{\prime}$ at $\theta_{e} \leq 1^{\circ}$, we assumed that the $L$ and $R$ components of $A$, $I_{L}^{(A)}$ and $I_{R}^{(A)}$, are proportional to difference spectra $I_{R}-I_{L}$. While we do not know the true polarization $P_{R}=\left\{I_{R}^{(A)}\right.$ $\left.-I_{L}^{(A)}\right\} /\left\{I_{R}^{(A)}+I_{L}^{(A)}\right\}, P_{R} \leq 0.6$ gives unphysical negative peaks. We subtracted the background $\frac{1}{2}\left\{I_{R}^{(A)}+I_{L}^{(A)}\right\}$, with different $P_{R}$ in the range 0.7-1.0, from the spectra in Fig. 1(b). This yielded the most probable peak positions and errors. These are plotted in Fig. 1(f) along with the band map obtained by ARPES, which indicates a band dispersing upward above bulk VBM toward $\bar{\Gamma}$ and reaches at $\sim 80 \mathrm{meV}$ at $k_{\|}$ $\leq 0.03 \AA^{-1}$. The feature is observed also in the ARPES data taken with He II $(40.8 \mathrm{eV})$, in agreement with the assignment of this band to a surface state.

Figure 3 shows the calculated states along $\bar{\Gamma} \bar{M}$. Thin lines indicate the bulk bands projected onto the (111) surface. The position of the bulk VBM is adjusted to the experiment. Note that there are bulk bands just above VBM as indicated by thin broken lines. As is well known, first-principles calculations based on density-functional theory underestimate band gaps. ${ }^{21}$ For Ge, the band gap is calculated to be zero at $\Gamma$ and at $L$. Note also that the SO split-off bulk band is seen below VBM with $\Delta_{\mathrm{SO}}=0.28 \mathrm{eV}$.

The calculated band structure exhibits spin-polarized states as indicated by circles. The spin polarization along $U / D$, as expected from Rashba SOI, is indicated in Fig. 3(a). 


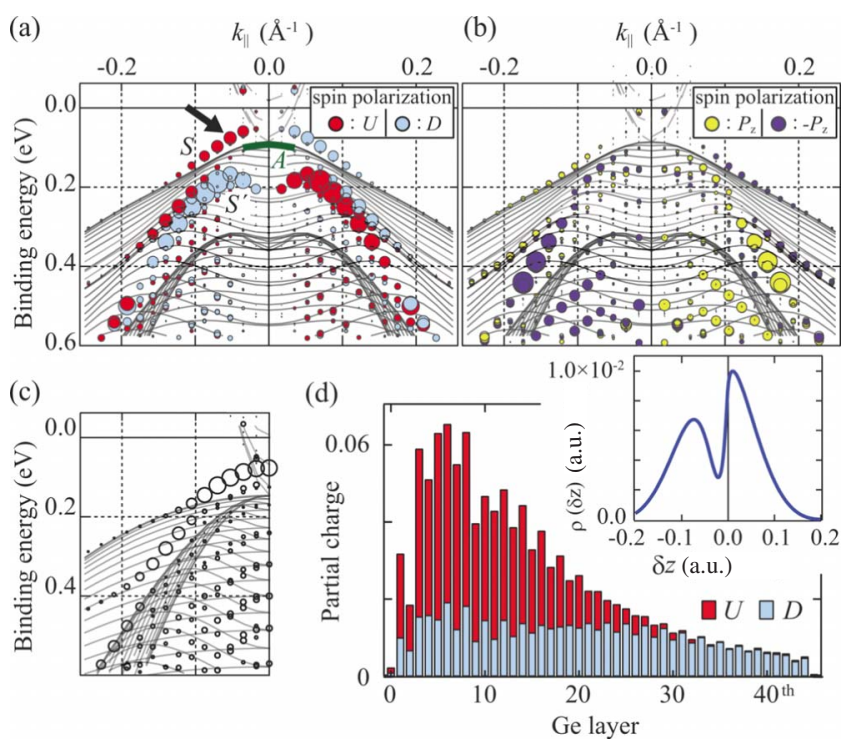

FIG. 3. (Color online) (a) Calculated band structure along $\bar{\Gamma} \bar{M}$ for the slab with $44 \mathrm{Ge}$ layers. The radii of the circles are proportional to the net contribution of Ge $4 p_{x} p_{y}$ from the first to 18th layers with spin oriented toward $U / D$. Thin lines indicate the projected bulk VB. (b) The same as (a) but with spin oriented along $z$. (c) The band structure calculated without SOC. The radii of the circles are proportional to the contribution of Ge $4 p_{x} p_{y}$ from the first to 18th layers. (d) Layer- and spin-resolved partial charges of the $S$ state at $k_{\|}=-0.052 \AA^{-1}$ indicated by an arrow in (a). The zeroth layer corresponds to the Bi adlayer. The inset shows chargedensity profile summed for the sixth- and seventh-layer Ge along $z$.

There are two spin-polarized bands, denoted as $S$, dispersing downward from $70 \mathrm{meV}$ at $\bar{\Gamma}$, and $S^{\prime}$ which has a maximum at $\sim 200 \mathrm{meV}$ at $k_{\|} \approx 0.05 \AA^{-1}$. $S$ disperses above the projected bulk bands, implying that this is a surface state. The layer-resolved partial charges of $S$ are shown in Fig. 3(d). The envelope of the charge-density distribution has a maximum at the sixth Ge layer and gradually decays into the bulk. While the $D$ component is less dependent on the depth from the surface, the $U$ component has a maximum amplitude at the fifth and sixth layers and mostly diminishes at the 30th layer. While the charge distribution of $S$ does not diminish at the opposite side of the slab, this must become zero at a finite depth for a slab with enough thickness since this state is located in the bulk band gap. The net spin polarization toward $D$ on the opposite side is due to Rashba SOI at the $\mathrm{H}$-terminated surface and hence should be considered as an artifact of the finite slab calculation. $S^{\prime}$ exhibits a similar depth dependence as $S$ and a finite amplitude at the H-terminated side. In principle, the resonance $S^{\prime}$ can have a finite amplitude in bulk while $S$ cannot. The difference would be only significant for thicker slabs.

The $S$ states have a negligible contribution from Bi adatoms, as demonstrated in Fig. 3(d), and are mostly of Ge $4 p_{x} p_{y}$ character with a much smaller contribution of $4 s$ and $4 p_{z}$ orbitals, where $z$ is defined to be parallel to surface normal. In order to show clearly the orbital character, the size of the circles in Fig. 3(a) is set proportional to the net contribution of Ge $4 p_{x} p_{y}$ from the first to 18th layers with
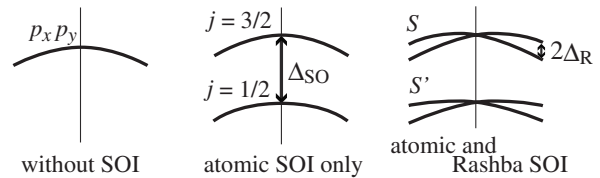

FIG. 4. Schematics for the roles of atomic and Rashba SOI.

spin oriented along $U / D$. Switching off the SOI only at Bi atoms makes negligible difference, ruling out also the scattering of bulk states at the surface Bi layer playing a role. ${ }^{22}$ These indicate that Bi SOI plays negligible role and the spin polarization of $S$ and $S^{\prime}$ is induced by SOI of Ge atoms.

In Fig. 3(c), the bands calculated without SOI are shown. Thin lines indicate the projected bulk bands calculated without SOI and hence the "split-off" bulk band is not split-off. A Ge-derived surface band is seen above the projected bulk bands, which is closely in accordance with the $S$ band. The $S^{\prime}$ band, however, has no corresponding band in the band structure without SOI. The comparison suggests a scenario (Fig. 4) similar to that for $\mathrm{Bi} / \mathrm{Ag}(111) .{ }^{23}$ First, the atomic SOI lifts the degeneracy of $p_{x}$ and $p_{y}$ at $\bar{\Gamma}$ and forms states that can be classified as $j=3 / 2(S)$ and $1 / 2\left(S^{\prime}\right)$ at $\bar{\Gamma}$. The energy difference of $0.18 \mathrm{eV}$ between $S$ and $S^{\prime}$ at $\bar{\Gamma}$ compares well with the atomic SO splitting of Ge $4 p(0.19 \mathrm{eV}) .{ }^{24}$ Second, at $k_{\|} \neq 0$, the $S$ and $S^{\prime}$ states are further split by Rashba SOI. Note that the lower branch of $S$ is seen as tiny dots in Figs. 3(a) and 3(b). For the $S$ band, the splitting $\Delta_{R}$ increases linearly up to $\sim 10 \mathrm{meV}$ at $k_{\|} \sim 0.05 \AA^{-1}$, yielding $\alpha_{R}$ $=0.2 \mathrm{eV} \AA$. The Rashba spin-split pair for $S^{\prime}$ cannot be specified unambiguously because of the hybridization with the bulk states.

The calculations done for the 10, 22, 32, and 44 layer slabs indicate the same spin polarization of the surface bands at almost the same binding energies. This indicates that these states are not quantum-well states, which must be shifted in energy depending on the slab thickness. The calculated binding energies and spin structure show a good agreement with those observed by SARPES.

The dispersion of $S$ and $S^{\prime}$ suggests the hybridization among two pairs of Rashba spin-split surface bands and bulk states. For $\left|k_{\|}\right|<0.1 \AA^{-1}$, the low-energy branch of $S$ shows the spin polarization parallel to that of the upper branch, though the magnitude is very small, and the $S$ and $S^{\prime}$ bands exhibit opposite spin polarization. These should be due to the hybridization between $S$ and $S^{\prime}$. Similar effect was found for the unoccupied bands on $\mathrm{Bi}$ and $\mathrm{Pb}$ on $\mathrm{Ag}$ (111) (Ref. 23) and was discussed in detail for $\mathrm{Bi} / \mathrm{Cu}(111) .{ }^{25}$ Smaller energy separation between $j=3 / 2$ and $1 / 2$ in the present case results in stronger hybridization. For $\left|k_{\|}\right|>0.15 \AA^{-1}$, the spin polarization of $S$ and $S^{\prime}$ toward $U / D$ is reduced. Spin polarization along $z$ [Fig. 3(b)] shows that the two bands get close in energy and their spins are reoriented toward the surface normal. At $\left|k_{\|}\right|>0.2 \AA^{-1}$, the spins of $S$ and $S^{\prime}$ are again oriented toward $U$ and $D$. Similar spin reorientation due to the hybridization was experimentally observed for $\mathrm{Bi}$ and $\mathrm{Pb}$ on $\operatorname{Ag}(111){ }^{26}$

As to the physical origin of the long-decaying Ge $p$-derived surface state, it should be noted that the band 
disperses in parallel with the edge of the bulk band with steeper dispersion as shown in Fig. 3(c). This suggests that the modification of potential due to the $\mathrm{Bi}$ adsorption should play important role in forming the surface band in a way similar to the well-formulated mechanism that yields socalled Shockley states. ${ }^{27}$ We confirmed by calculation that substitution of $\mathrm{Bi}$ with other elements such as $\mathrm{Ag}$ yields similar surface bands.

The magnitude of Rashba SOI is determined by the asymmetry of charge distribution along the surface normal at closest proximity to atomic nuclei because of the contribution of the steepest nuclear potential gradient. ${ }^{14}$ For a bulk Ge atom, while the charge distribution is asymmetric along the [111] direction, the asymmetry cancels out for adjacent layers. The inset in Fig. 3(d) shows the charge distribution along the surface normal summed over the sixth and seventh layer Ge atoms, at which amplitude and spin polarization are largest, as a function of relative $z$ coordinate, $\delta z$, with respect to the nuclei, indicating a peak at $\delta z \sim 0.01$ a.u. and a dip at $\delta z$ $\sim-0.02$ a.u. (1 a.u. $=0.529 \AA)$, which contribute to Rashba SOI.

In summary, we have found Ge $4 p$-derived surface bands on the Bi-covered Ge(111) surface. These are split by atomic
SOI at $\bar{\Gamma}$, resulting in a surface-state band and a surfaceresonance band. The both bands are spin polarized due to Rashba SOI. In order to realize spin current at semiconductor surfaces, a metallic, spin-polarized band is of crucial importance. ${ }^{10}$ Although the spin-polarized $S$ and $S^{\prime}$ bands on $\mathrm{Bi} / \mathrm{Ge}(111)$ are not metallic, a small amount of hole doping would make the $S$ band metallic. Since there are no other metallic bands on this surface, the surface conductivity ${ }^{28}$ should be governed by $S$, which should then give rise to spin-polarized current and work as a spin filter. ${ }^{29}$ An important point is that such a long-decaying surface state with small or negligible amplitude at the topmost surface layer is essentially an interface state and can be induced at any heterojunctions. Similar spin-polarized states should be obtained at Ge surfaces covered with thicker films of appropriate materials, which can then be used for various transport experiments under atmospheric conditions.

The authors thank K. Kanomaru for his technical support in the SARPES measurement at HSRC, which was done under the approval by Proposal Assessing Committee of HSRC (Proposal No. 09-A-30). *aruga@kuchem.kyoto-u.ac.jp

${ }^{1}$ É. I. Rashba, Sov. Phys. Solid State 2, 1109 (1960); Yu. A. Bychkov and É. I. Rashba, JETP Lett. 39, 78 (1984).

${ }^{2}$ J. H. Dil J. Phys.: Condens. Matter 21, 403001 (2009).

${ }^{3}$ S. LaShell, B. A. McDougall, and E. Jensen, Phys. Rev. Lett. 77, 3419 (1996).

${ }^{4}$ J. Henk et al., J. Phys.: Condens. Matter 16, 7581 (2004).

${ }^{5}$ Y. M. Koroteev, G. Bihlmayer, J. E. Gayone, E. V. Chulkov, S. Blügel, P. M. Echenique, and P. Hofmann, Phys. Rev. Lett. 93, 046403 (2004).

${ }^{6}$ T. Nakagawa, O. Ohgami, Y. Saito, H. Okuyama, M. Nishijima, and T. Aruga, Phys. Rev. B 75, 155409 (2007).

${ }^{7}$ C. R. Ast, J. Henk, A. Ernst, L. Moreschini, M. C. Falub, D. Pacilé, P. Bruno, K. Kern, and M. Grioni, Phys. Rev. Lett. 98, 186807 (2007).

${ }^{8}$ I. Gierz, T. Suzuki, E. Frantzeskakis, S. Pons, S. Ostanin, A. Ernst, J. Henk, M. Grioni, K. Kern, and C. R. Ast, Phys. Rev. Lett. 103, 046803 (2009).

${ }^{9}$ S. Hatta, T. Aruga, Y. Ohtsubo, and H. Okuyama, Phys. Rev. B 80, 113309 (2009).

${ }^{10}$ K. Yaji, Y. Ohtsubo, S. Hatta, H. Okuyama, K. Miyamoto, T. Okuda, A. Kimura, H. Namatame, M. Taniguchi, and T. Aruga, Nature Commun. 1, 17 (2010).

${ }^{11}$ S. A. Wolf et al., Science 294, 1488 (2001).

${ }^{12}$ D. D. Awschalom and M. E. Flatté, Nat. Phys. 3, 153 (2007).

${ }^{13}$ K. Sugawara, T. Sato, S. Souma, T. Takahashi, M. Arai, and
T. Sasaki, Phys. Rev. Lett. 96, 046411 (2006).

${ }^{14}$ M. Nagano et al., J. Phys.: Condens. Matter 21, 064239 (2009).

${ }^{15}$ Y. Ohtsubo et al., J. Phys.: Condens. Matter 21, 405001 (2009).

${ }^{16}$ P. Blaha et al., WIEN2k: An Augmented Plane Wave + Local Orbitals Program for Calculating Crystal Properties (Karlheinz Schwarz, Techn. Universität Wien, Wien, 2001).

${ }^{17}$ M. Perego et al., J. Appl. Phys. 100, 093718 (2006).

${ }^{18}$ A. L. Wachs, T. Miller, T. C. Hsieh, A. P. Shapiro, and T. C. Chiang, Phys. Rev. B 32, 2326 (1985).

${ }^{19}$ A. Fanelsa, E. Kisker, J. Henk, and R. Feder, Phys. Rev. B 54, 2922 (1996).

${ }^{20}$ E. Tamura, W. Piepke, and R. Feder, Phys. Rev. Lett. 59, 934 (1987).

${ }^{21}$ A. J. Freeman et al., in Solid-State Photoemission and Related Methods, Theory and Experiment, edited by W. Schattke and M. A. Van Hove (Wiley, Berlin, 2003), p. 1.

${ }^{22}$ A. Kimura et al., Phys. Rev. Lett. 105, 076804 (2010).

${ }^{23}$ G. Bihlmayer, S. Blügel, and E. V. Chulkov, Phys. Rev. B 75, 195414 (2007).

${ }^{24}$ S. Kotochigova et al., Phys. Rev. A 55, 191 (1997).

${ }^{25}$ H. Mirhosseini et al., Phys. Rev. B 79, 245428 (2009).

${ }^{26}$ F. Meier et al., Phys. Rev. B 77, 165431 (2008).

${ }^{27}$ M. C. Desjonquères and D. Spanjaard, Concepts in Surface Physics, 2nd ed. (Springer, New York, 1996).

${ }^{28}$ S. Hasegawa et al., Surf. Rev. Lett. 10, 963 (2003).

${ }^{29}$ F. Meier et al., Phys. Rev. B 79, 241408(R) (2009). 